\title{
High rates of recurrence of depression in children and adolescents
}

Dunn V, Goodyer IM. Longitudinal investigation into childhood and adolescence-onset depression: psychiatric outcome in early adulthood. Br J Psych 2006; 188:216-22.

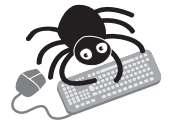

This article contains extra text on the EBMH website

\section{What is the long term prognosis for children with depression in clinic and community samples, and what are the} prognostic factors?

\section{METHODS}

$-4$

Design: Prospective cohort study.

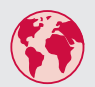

Setting: Cambridgeshire, UK; recruitment 1991-96.

की

Population: Eighty three children and adolescents aged 8-17 years with depression. Clinic group: 58 participants aged 8-16 years attending a mental health clinic with depression (DSM-III-R major depression, minor depression, or dysthymia, assessed using the Schedule for Affective Disorders and Schizophrenia for School-Age Children (K-SADS), Present version). Community group: 25 participants aged 12-17 years with depression (DSMIII-R, assessed using the K-SADS, Present and Lifetime version), identified from local schools.

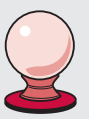

Prognostic factors: Gender, pubertal stage at onset of depression, self-reported Mood and Feeling Questionnaire (MFQ) score, age at onset of index episode, severity of impairment, duration of depression before treatment, age at onset of first episode.

Fivell

Outcomes: Full remission of depression ( $\geqslant 2$ months without depressive symptoms or functional impairment), recurrence of depression after full remission (Structured Clinical Interview for DSM-IV Axis I disorders (SCID-I)).

Follow up period: Until age 22 years. Overall mean follow up 7.8 years (clinic group: 8.1 years; control group: 9.2 years; community group: 5.8 years; $p<0.001$ for difference).

\section{MAIN RESULTS}

The clinic group had more indicators of severe depression at baseline than the community group (see http://www.ebmentalhealth.com/ supplemental for table). Full remission from the index episode occurred in $78 \%$ of the clinic group and $92 \%$ of the community group. Median time to remission from the index episode was 24 months in the clinic group and 3 months in the community group $(\mathrm{p}<0.001)$. The prognostic factors associated with longer index episodes in the clinic group were more severe impairment during the index episode (HR 3.27, 95\% CI 1.60 to 6.65), longer periods of depression before treatment (HR $0.95,95 \%$ CI 0.92 to 0.99 ), and younger age at first psychiatric episode (HR 1.12, 95\% CI 1.00 to 1.24). In the clinic group, $44 \%$ of those who remitted had a recurrence, compared with $52 \%$ in the community group (significance not reported). The median time to first recurrence was 9.25 years in the clinic group, and mean time to first recurrence in the community group was 3.4 years (median not reported). The main prognostic factor associated with reduced time to recurrence in the clinic group was female gender (HR 5.4, 95\% CI 1.1 to 25.7$)$. In $22 \%$ of children and adolescents in the clinic group and in $8 \%$ of the community group, the index episode persisted until age 22 years.

For correspondence: Valerie Dunn, Developmental Psychiatry Section, Department of Psychiatry, University of Cambridge, Cambridge, UK; vjd20@cam.ac.uk

Source of funding: Wellcome Trust.

\section{CONCLUSIONS}

Recurrent depression is common in children and adolescents with depression identified at mental health clinics and in the community. Greater severity of index episode, earlier onset, and longer duration of illness before treatment are associated with increased time to remission of the index episode. Female gender is associated with earlier recurrence.

\section{NOTES}

The study also included a control group without depression that is not reported here. Loss to follow up from the original clinic sample ( 120 participants) was $45 \%$, compared with $29 \%$ from the community sample (35 participants). Dropouts from the clinic group had significantly different characteristics to those who participated in follow up (such as younger age at onset of index episode).

\section{Commentary}

D unn and Goodyer's study, which well represents the next step towards investigating the long term outcome of school age depression in clinic and community samples, highlights that early onset depression often represents the beginning of a lifelong course of depression.

Lifetime prevalence rates of DSM-III-R major depression ${ }^{2}$ increase in a linear trajectory from preadolescence to adolescence. Specifically, major depressive disorder (MDD) lifetime prevalence rates begin at $3 \%$ at age 13 years, surge to $14.6 \%$ by age 15 years, and swell to $21.2 \%$ by age 21 years. ${ }^{3}$ Also, considerable evidence now demonstrates that MDD is a recurrent condition with a cumulative probability of recurrence of $40 \%$ by 2 years and $70 \%$ by 5 years of first episode. ${ }^{4}$

Dunn and Goodyer consider the effects of gender, severe impairment at index of depression, experiencing depression long before starting treatment, and an early age of first onset of depression. Such investigations may significantly improve our understanding regarding the patterns of symptoms that can be ascribed to recurrent depression. Ultimately, greater understanding of these risk factors will help to clarify the trajectories of depressive symptoms over time. The authors remind us that the potential for unremitting affective illness justifies the initiation of early, extended, and exhaustive clinical intervention, with regular follow up sessions to prevent relapse. We suggest that clinical investigation of these risk factors using a developmental framework ${ }^{5}$ may develop our appreciation for how developmental tasks modify the expression of depression in adolescents. Conversely, depression influences the competencies and developmental tasks facing emerging adults that may potentially increase the risk of recurrence of affective illness. Jackie K Gollan, PhD and Heather Pane, MPP

Department of Psychiatry, University of Chicago, Chicago, IL, USA

1 Gollan JK, Raffety B, Gortner ET, et al. Course profiles of early and adult onset of depression. J Affect Dis 2005;86:81-6.

2 American Psychiatric Association. Diagnostic and Statistical Manual of Mental Disorders (4th edition). Washington DC: American Psychiatric Association Press, 1994.

3 Birmaher B, Ryan ND, Williamson DE, et al. Childhood and adolescent depression: A review of the past 10 years-part I. J Am Acad Child Adol Psych 1996;35:1427-39.

4 Insel TR, Nakamura RK. Breaking Ground, Breaking Through: the strategic plan for mood disorders research of the National Institute of Mental Health. National Institute of Mental Health, 2002:10.

5 Cicchetti D, Toth S. The development of depression in children and adolescents. Am Psychol 1988;74:221-41. 